\title{
Terapia de presión negativa en pediatría
}

\author{
Negative pressure therapy in pediatrics
}

\author{
Luis Mauricio Figueroa-Gutiérrez $\mathbb{D}^{\mathbb{D}}$, Jaime Martínez-Cano ${ }^{2} \mathbb{D}$, Beatriz Giraldo-Ossa ${ }^{3} \mathbb{D}$, \\ Luz Adriana López-González $\mathbb{D}^{\mathbb{D}}$, Silvio Echeverry-Rendón ${ }^{5} \mathbb{D}$, Eliana Rodríguez-Suárez $\mathbb{D}^{5}$, \\ Sebastián Galvis-Acevedo ${ }^{5}$ D, Jhonatan Augusto Flórez-López ${ }^{5} \mathbb{D}$
}

\footnotetext{
Médico, especialista en Cirugía general y Cirugía pediátrica; docente, Universidad del Valle, Cali, y Universidad Tecnológica de Pereira, Pereira, Colombia

2 Médico, especialista en Cirugía general y Cirugía pediátrica; Clínica Comfamiliar, Pereira, Colombia

3 Enfermera, especialista en Terapia Enterostomal; Clínica Comfamiliar, Pereira, Colombia

4 Enfermera, especialista en Pedagogía para Docencia Universitaria y en Gerencia de la Calidad y Auditoría en Salud; docente, Universidad Libre Seccional Pereira, Pereira, Colombia

5 Médico, Universidad Tecnológica de Pereira, Colombia
}

\section{Resumen}

Introducción. La terapia de presión negativa es un recurso utilizado cada vez con mayor frecuencia en el manejo de heridas complejas en pediatría. El objetivo de este estudio fue describir la experiencia con esta terapia en diferentes situaciones clínicas.

Métodos. Se llevó a cabo un estudio descriptivo retrospectivo en un grupo de pacientes pediátricos en quienes se utilizó la terapia de presión negativa entre el año 20IO y el 2015. Se analizaron las variables sociodemográficas, los diagnósticos que indicaron la terapia, el tiempo de uso, sus complicaciones y la mortalidad.

Resultados. Se incluyeron 4I pacientes. La terapia se indicó en 39 casos con heridas localizadas en el abdomen, en uno con infección de los tejidos blandos perianales y en otro con una herida de esternotomía infectada. De las heridas abdominales, I4 fueron por complicaciones relacionadas con apendicitis aguda, 6 por enfermedades relacionadas con megacolon, 5 por obstrucción intestinal, 4 para el manejo de fístulas, 4 por enterocolitis necrosante del recién nacido, 3 por pancreatitis aguda y 3 por otras causas. El tiempo promedio de uso de la terapia fue de 7 días. Se presentaron fallas en el sistema de vacío en dos pacientes, pero no hubo complicaciones por el uso de la terapia. Dos pacientes fallecieron por complicaciones relacionadas con su enfermedad de base.

Conclusión. La terapia de presión negativa es un recurso efectivo en el manejo de heridas complejas en la población pediátrica.

Palabras clave: terapia de presión negativa para heridas; vacío; apendicitis; enterocolitis necrotizante; pediatría.

Fecha de recibido: 30/09/2019 - Fecha de aceptación: 16/10/2019

Correspondencia: Luis Mauricio Figueroa-Gutiérrez, Carrera 19 \# 94-96 Villasol Manzana B casa 19, Pereira, Colombia.

Teléfono: 313-7371166

Correo electrónico: Imfg13@utp.edu.co

Citar como: Figueroa-Gutiérrez LM, Martínez-Cano J, Giraldo-Ossa B, López-González LA, Echeverry-Rendón S, et al. Terapia de presión negativa en pediatría. Rev Colomb Cir. 2020;35:614-20. https://doi.org/10.30944/20117582.483

Este es un artículo de acceso abierto bajo una Licencia Creative Commons - BY-NC-ND https://creativecommons.org/licenses/by-ncnd/4.0/deed.es 


\begin{abstract}
Introduction. Negative pressure therapy is an increasingly used resource in the management of complex wounds in pediatrics. The objective of this study was to describe the experience with this therapy in different clinical situations.

Methods. A retrospective descriptive study was conducted in a group of pediatric patients who received negative pressure therapy between 2010 and 2015. We analyzed the sociodemographic variables, the diagnoses that indicated the therapy, the time of use of the therapy, complications and mortality.

Results. A total of $4 \mathrm{I}$ patients were included. Therapy was indicated in 39 cases with wounds located in the abdomen, in one with infection of the perianal soft tissues, and in another with an infected sternotomy wound. Of the abdominal wounds, I4 were due to complications related to acute appendicitis, six due to diseases related to megacolon, five due to intestinal obstruction, four for the management of fistulas, four due to necrotizing enterocolitis of the newborn, three due to acute pancreatitis, and three due to other causes. The average time of use of the therapy was 7 days. Vacuum system failures occurred in two patients, but there were no complications from the use of therapy. Two patients died of complications related to their underlying disease.
\end{abstract}

Conclusion. Negative pressure therapy is an effective resource in the management of complex wounds in the pediatric population.

Keywords: negative-pressure wound therapy; vacuum; appendicitis; enterocolitis, necrotizing; pediatrics.

\section{Introducción}

El concepto de presión negativa para el manejo de heridas quirúrgicas complejas fue introducido por primera vez en 1993 por Fleishmann, quien desarrolló un sistema para el tratamiento de fracturas abiertas ' . En 1997, Argenta y Morykwas presentaron los primeros resultados del cierre asistido con vacío (vaccum-assisted closure, VAC) en el manejo de heridas complejas diversas, demostrándose sus beneficios en lo que respecta a la promoción de la angiogénesis, el desarrollo de tejido de granulación y la disminución del edema entre otras cualidades, con un resultado favorable en el $98 \%$ de los casos ${ }^{2,3}$. La terapia se fue popularizando en adultos ${ }^{4-7} \mathrm{y}$, en el 2005, Caniano, et al., reportaron los primeros casos en población pediátrica utilizando este sistema con resultados favorables en lesiones de tejidos blandos de origen infeccioso y traumático, en las extremidades y en enfermedades abdominales de diferente tipo ${ }^{8}$. De este modo, las experiencias con este tipo de terapia fueron aumentando, incluso, en enfermedades propias de neonatos prematuros ${ }^{9-13}$.
En el presente trabajo se describe la experiencia con la terapia de presión negativa en población pediátrica en diferentes situaciones clínicas.

\section{Métodos}

Se llevó a cabo un estudio descriptivo y retrospectivo, entre el 2010 y el 2015, en pacientes menores de I4 años a quienes, por las características especiales de sus heridas, se les indició tratamiento con terapia de presión negativa.

Las variables que se analizaron fueron: características sociodemográficas (sexo, edad), los diagnósticos que indicaron la terapia, la ubicación anatómica de las heridas (abdomen, tejidos blandos, tórax), la magnitud de la presión utilizada, el número de recambios del sistema, las complicaciones derivadas de la terapia y la mortalidad. Los datos se presentan como medias, rangos y medianas.

Para el presente estudio, se utilizó un sistema de terapia de presión negativa convencional (figura I). Este consta de una película plástica con múltiples perforaciones o un apósito de polivinilo que se coloca directamente en contacto con 

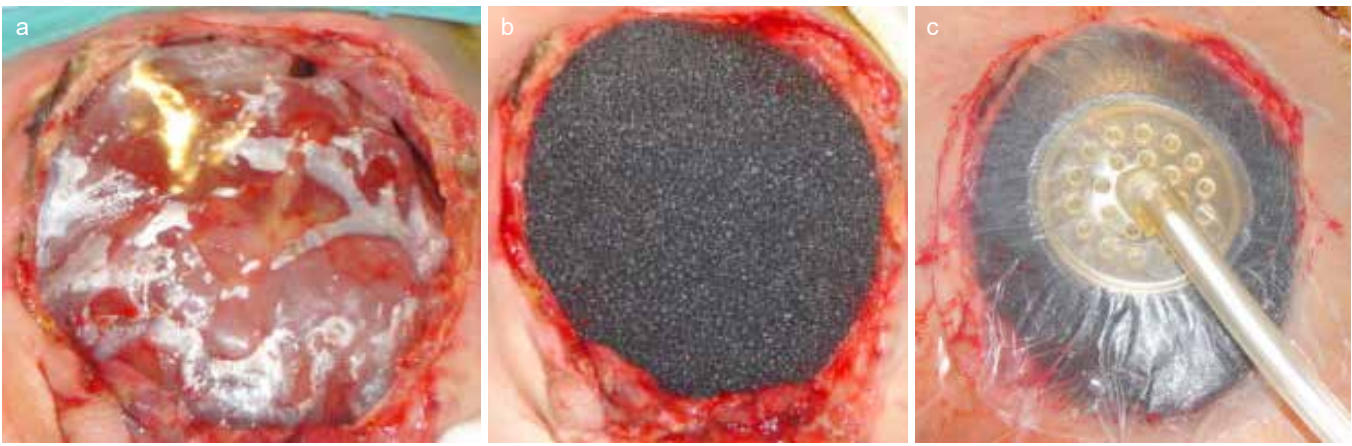

Figura 1. a. Película plástica con múltiples perforaciones. b. Apósito de poliuretano. c. Adhesivo transparente y sistema de vacío

las asas intestinales, los tendones y los orificios fistulosos, entre otros. Sobre esto, se coloca una esponja de poliuretano, la cual se adapta al tamaño de la lesión o herida. Estos componentes se recubren con un apósito adhesivo transparente que se comunica con una unidad eléctrica generadora de vacío o presión negativa que varía entre -50 y -IOO mm Hg.

Los recambios del sistema se programan según la necesidad o para un tiempo no mayor de 96 horas; además, se revisan las condiciones de las estructuras involucradas y las necesidades de desbridamiento, de lavado o de reconstrucción.

Según cada caso en particular, se decide si se reinstala o no el sistema, si se retira y se cierra la herida, o si se continua con otro tipo de tratamiento.

\section{Resultados}

Se incluyeron 4I pacientes, de los cuales 25 (6I \%) eran de sexo masculino, con una media de edad de 7 años (rango: o a I4 años) (tabla I). En 39 (95\%) de los casos, la terapia de presión negativa se indicó en heridas localizadas en el abdomen, en un caso $(2,4 \%)$, en los tejidos blandos perianales (figura 2), y en otro, en una herida de esternotomía infectada (figura 3). Este último requirió cirugía cardiaca y traqueostomía, pero se consiguió la resolución completa, sin pérdida del esternón o inestabilidad ósea (figura 4).

La mediana del tiempo de uso de la terapia de presión negativa fue de 4 días y la media de
Tabla 1. Aspectos demográficos e indicación de la terapia de presión negativa

\begin{tabular}{|c|c|c|c|}
\hline Variables & Descripción de la muestra & $\mathbf{n}$ & $\%$ \\
\hline \multirow{4}{*}{ Edad } & $\begin{array}{l}\text { Prematuro (<37 semanas de } \\
\text { gestación) }\end{array}$ & 4 & 9,7 \\
\hline & $\begin{array}{l}\text { Neonato ( } 38 \text { semanas de } \\
\text { gestación) - } 12 \text { meses }\end{array}$ & 2 & 4,6 \\
\hline & 1-5 años & 14 & 34,1 \\
\hline & 6-14 años & 21 & 51,2 \\
\hline \multirow{2}{*}{ Sexo } & Hombres & 25 & 60,9 \\
\hline & Mujeres & 16 & 39 \\
\hline \multirow{15}{*}{ Indicación } & Abdomen & & \\
\hline & Enterocolitis necrosante & 4 & 9,7 \\
\hline & Apendicitis aguda & 14 & 34,1 \\
\hline & Fístulas & 4 & 9,7 \\
\hline & Obstrucción intestinal (bridas) & 5 & 12,2 \\
\hline & Megacolon & 6 & 14,6 \\
\hline & Hemoperitoneo por dengue & 1 & 2,3 \\
\hline & Traumatismo hepático & 1 & 2,3 \\
\hline & Invaginación & 1 & 2,3 \\
\hline & Pancreatitis aguda & 3 & 7,3 \\
\hline & Tórax & & \\
\hline & Osteomielitis esternal & 1 & 2,3 \\
\hline & Tejidos blandos & & \\
\hline & Región perianal & 1 & 2,3 \\
\hline & Total & 41 & 100 \\
\hline
\end{tabular}

7,7 días. En 4 (9,7 \%) de las heridas abdominales hubo necesidad de reiniciarla por una nueva causa o por complicaciones de la enfermedad de base. De los 4I pacientes, I8 (43,9\%) requirieron la instalación del sistema por una única vez; I7 $(4 \mathrm{I}, 4 \%)$, un recambio; I $(2,4 \%)$, dos recambios, 
y 5 (I2,2 \%), tres recambios como máximo. Las presiones utilizadas variaron según la edad, el peso y las condiciones del paciente, y fluctuaron entre -50 y - $100 \mathrm{~mm} \mathrm{Hg}$.

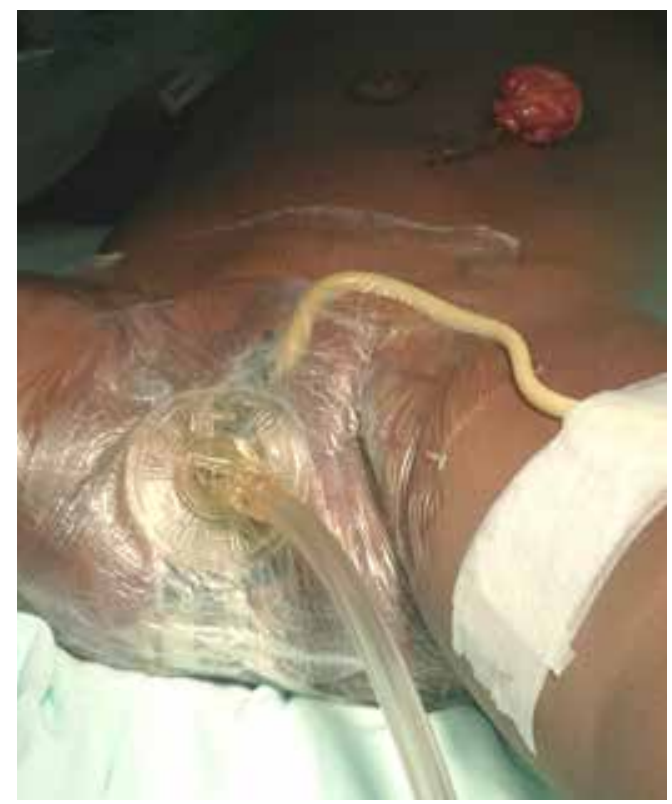

Figura 2. Sistema instalado en infección de tejidos blandos perianal.

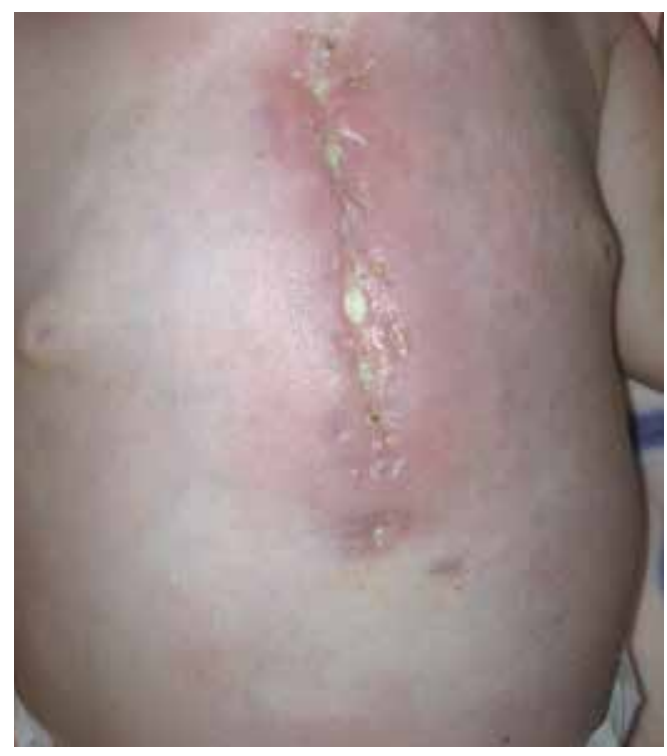

Figura 3. Infección de esternotomía.
No se presentaron complicaciones graves derivadas de la terapia de presión negativa. Sin embargo, en dos $(4,6 \%)$ casos ocurrieron disfunciones del sistema de vacío por fugas que fueron corregidas sin problema y, en todos los casos, dicha terapia favoreció el cierre de una herida compleja. Finalmente, se presentaron 4 (9,7\%) fallecimientos no asociados con la terapia de presión negativa, causados uno por un linfoma intestinal perforado, otro por sepsis originada por perforación del colon, otro por trauma hepático y otro por dengue hemorrágico.

\section{Discusión}

Se reconocen múltiples mecanismos físicos y biológicos mediante los cuales actúa la terapia de presión negativa: reduce el edema local, influye en el proceso inflamatorio por medio del reclutamiento de fibroblastos, induce la migración celular, reduce la población bacteriana al debilitar los procesos enzimáticos bacterianos, modifica el medio ambiente de la herida, reduce la exposición de la herida a los líquidos de drenaje e induce un proceso de cicatrización centrípeto. Al promover la angiogénesis y la formación de tejido de granulación, esta terapia favorece

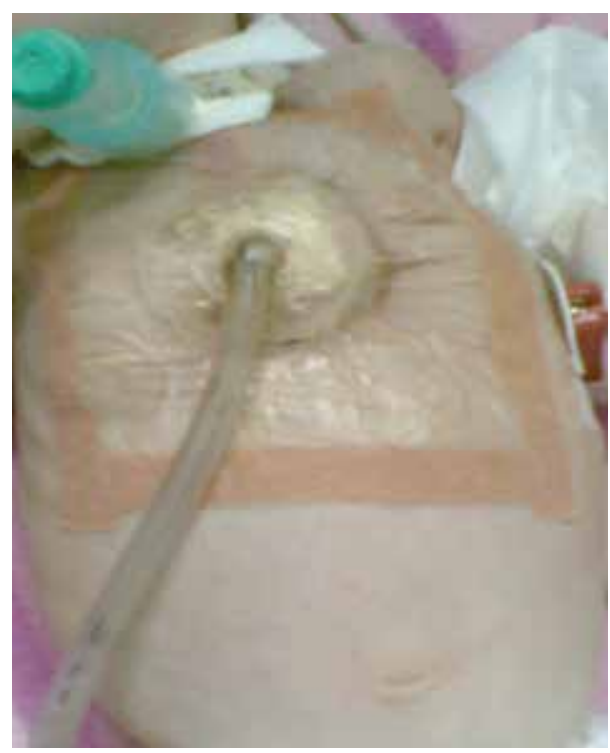

Figura 4. Sistema de presión negativa instalado en una herida del esternón 
la aproximación de los bordes de la herida y el cierre primario, secundario o terciario de la herida ${ }^{14,15}$. Puede usarse sola o asociada con otros apósitos complementarios para facilitar el cierre definitivo de la herida (cierre directo de la herida abdominal después de controlar el síndrome de compartimiento o la sepsis, la instalación de prótesis, los injertos o los colgajos) o inducir la cicatrización por segunda intención ${ }^{16}$.

A pesar de sus probadas ventajas y cualidades, y la relativa gran frecuencia en pediatría de heridas complejas, síndrome de compartimiento y sepsis abdominal, la terapia de presión negativa tiene un uso excepcional en los departamentos de cirugía pediátrica, en comparación con las unidades de cirugía plástica, ortopédica y de cirugía general de adultos ${ }^{17}$. Esto puede atribuirse a la limitada exposición de los cirujanos pediatras a este método y la poca difusión de la evidencia científica de su eficacia y seguridad en la población pediátrica. Muchos casos pediátricos se han reportado en series de adultos en publicaciones de cirugía plástica, ortopédica y cardíaca. No obstante, la disponibilidad de series de casos exclusivamente pediátricos es escasa ${ }^{16}$.

En el 2005, se publicó la primera serie de casos sobre la terapia de presión negativa en pacientes pediátricos con compromiso de tejidos blandos, extremidades o abdomen abierto. En ella se concluyó que dicha terapia es una alternativa terapéutica útil para el manejo de heridas complejas en niños, debido a su seguridad, costo-efectividad y ventajas, como menor frecuencia de cambio de apósitos, posibilidad de tratamiento ambulatorio de algunos casos y gran aceptación y tolerancia por parte del paciente ${ }^{8}$.

El número de reportes sobre la terapia de presión negativa en todos los grupos etarios pediátricos ha venido aumentando ${ }^{9-13,16} \mathrm{y}$, específicamente en los neonatos, a partir de las primeras publicaciones en el $2005^{8,9,18}$. Más adelante, se comprobó su utilidad en catástrofes abdominales por enterocolitis necrosante, en lesiones complejas por necrosis de la pared abdominal, en dehiscencias de anastomosis e infecciones esternales, entre otros, obteniéndose resultados alentadores para este grupo de edad ${ }^{19}$.
En el presente trabajo, los casos de neonatos con enterocolitis necrosante se abordaron con una primera intervención quirúrgica que consistió en una cirugía de control de daños (drenaje y manejo de peritonitis generalizada, resección intestinal, cierre con ligadura de las bocas sin anastomosis y laparostomía), instalación del sistema de terapia de presión negativa y posteriores revisiones. Esto permitió practicar una anastomosis intestinal sin complicaciones y evitar la necesidad de ostomías, las cuales, por sus pérdidas intestinales de alto gasto, generan riesgo de deshidratación y no están exentas de complicaciones. Al final, se obtuvo un resultado satisfactorio, similar al que se ha reportado con este abordaje en diferentes publicaciones ${ }^{20-22}$.

En adultos se han reportado las complicaciones asociadas con el sistema. En niños, Rentea, et al. ${ }^{23}$, presentaron los resultados de una serie de 290 pacientes atendidos en un período de cuatro años, en quienes se utilizó la terapia de presión negativa y se reportando las siguientes complicaciones en 5 ( $\mathrm{I}, 7 \%$ ) casos: formación de una fístula entero-atmosférica, retención de la esponja de poliuretano y reinfección de la herida, cada una en un caso, y laceración de la piel por el adhesivo en los otros dos casos. En el presente estudio, el sistema de vacío falló en dos pacientes, lo que se resolvió reinstalando el sistema, y no se presentaron complicaciones atribuibles a la terapia en sí.

Los niveles de presión negativa utilizados en la población pediátrica han sido tema de discusión en las diferentes series de casos reportadas. Sin embargo, existe un acuerdo sobre los límites de los rangos de presión utilizados. En el 2009, con base en las primeras recomendaciones de expertos, se aconsejó una presión entre -50 y -75 $\mathrm{mm} \mathrm{Hg}$ para los niños menores de dos años y, una de -75 a -I25 mm Hg, para los mayores de I2 años. Para aquellos entre 2 y I2 años de edad, se recomendó modificar la presión, dentro de estos mismos rangos, según la localización de la herida; por ejemplo, en heridas esternales, la presión negativa debe oscilar entre -50 y $-75 \mathrm{~mm}$ $\mathrm{Hg}$ en todos los grupos etarios pediátricos ${ }^{24}$. Es- 
tas recomendaciones fueron tenidas en cuenta en el presente estudio.

A pesar de las advertencias sobre la terapia de presión negativa en la población pediátrica que ha emitido la Food and Drug Administration (FDA), existen situaciones en las cuales no se dispone de otra mejor alternativa terapéutica ${ }^{23,25}$. Por ello, se considera que esta terapia es un recurso útil, efectivo, de manejo seguro, que debe tenerse en cuenta para tratar heridas complejas en niños de todos los grupos de edad. Ha demostrado buenos resultados y bajas tasas de complicaciones, sin poner en riesgo la seguridad y la vida del paciente, siempre que se cumpla con la estandarización de los protocolos de uso y la capacitación del personal al cuidado del paciente.

En diferentes estudios, la terapia de presión negativa ha demostrado ser costo-efectiva en el tratamiento de heridas complejas, lo cual se refleja en el ahorro de insumos y dispositivos médicos, la disminución de los días de hospitalización, el manejo domiciliario en casos seleccionados y la reducción del número de curaciones necesarias para agilizar el cierre y la cicatrización ${ }^{15,26}$.

Por último, en Colombia, aunque la utilidad de la terapia de presión negativa en cirugía pediátrica en diferentes instituciones de salud ha sido muy difundida, solo existen reportes de experiencias con su uso en adultos ${ }^{27-29}$, por lo cual este estudio se convierte en un importante referente para otras investigaciones y aplicaciones de esta terapia en heridas complejas en población pediátrica en el país.

\section{Cumplimiento de normas éticas}

Consentimiento informado. Se obtuvo el consentimiento informado por parte de los padres de los pacientes para las intervenciones quirúrgicas, la participación en publicaciones y la toma de fotografías. El comité de ética institucional aprobó el diseño y la metodología del estudio.

Declaración de conflicto de intereses. Los autores declaran que no tienen conflicto de intereses.

Fuentes de financiación: recursos propios de los autores.
Agradecimientos: Al epidemiólogo y asesor en investigación clínica, Jorge Mario Estrada Álvarez, por su colaboración y apoyo en la elaboración de este trabajo.

\section{Referencias}

I. Fleichsmann W, Strecker W, Bombelli M, Kinzl L. Vaccum sealing as treatment of soft tissue damage in open fractures. Unfallchirurg. 1993;96:488-92.

2. Argenta L, Morykwas M. Vacuum-assisted closure: A new method for wound control and treatment: Clinical Experience. Ann Plast Surg. 19970;38:563-76. https://doi.org/I0.I097/00000637-199706000-00002

3. Morykwas M, Argenta L, Shelton-Brown E, McGuirt W. Vacuum-assisted closure: A new method for wound control and treatment: animal studies and basic foundation. Ann Plast Surg. 1997;38:553-62. https://doi.org/I0.I097/00000637-199706000-0000I

4. Joseph E, Hamori CA, Bergman S, Roaf E, Swann NF, Anastasi GW. Prospective randomized trial of vacuum-assisted closure versus standard therapy of chronic non-healing wounds. Wounds. 2000;12:60-7.

5. DeFranzo AJ, Argenta LC, Marks MW, Molnar JA, David $L R$, Webb $L$, et al. The use of vacuum assisted closure therapy for the treatment of lower-extremity wounds with exposed bone. Plast Reconstr Surg. 200I;I08:II849I. https://doi.org/I0.I097/00006534-200110000-00013

6. Herscovici D Jr, Sanders R, Scaduto J, Infante A, DiPasquale T. Vacuum-assisted wound closure (V.A.C. therapy) for the management of patients with high-energy soft tissue injuries. J Orthop Trauma. 2003;17:683-8. https://doi.org/I0.I097/0000513I-200311000-00004

7. Parrett B, Matros E, Pribaz JJ, Orgill DP. Lower extremity trauma: Trends in the management of soft-tissue reconstruction of open tibia-fibula fractures. Plast Reconstr Surg. 2006;117:1315-22. https://doi.org/IO.I097/oI.prs.0000204959.I8I36.36

8. Caniano D, Ruth B, Teich S. Wound management with vacuum-assisted closure: Experience in 5I pediatric patients. J Pediatr Surg. 2005;40:I28-32.

https://doi.org/IO.IOI6/j.jpedsurg.2004.09.016

9. Fleck T, Simon P, Burda G, Wolner E, Wollenek G. Vaccum assisted closure therapy for the treatment of sternal wound infections in neonates and small infants. Interact Cardiovasc Thorac Surg. 2006;5:285-8.

https://doi.org/IO.I5Io/icvts.2005.I22424

Io. López G, Clifton-Koeppel R, Emil S. Vacuum-assisted closure for complicated neonatal abdominal wounds. J Pediatr Surg. 2008;43:2202-7. https://doi.org/Io.IOI6/j.jpedsurg.2008.08.067

II. Sorolla J, Cabello R, Fontbona M, Dagnino B, Obaíd $\mathrm{M}$, Giugliano C. Experiencia en manejo de heridas y 
defectos de pared abdominal con sistema de presión negativa continua en recién nacidos. Revista Chilena de Cirugía Plástica. 2010;8:57-62.

12. Stoffan AP, Ricca R, Lien C, Quigley S, Linden BC. Use of negative pressure wound therapy for abdominal wounds in neonates and infants. J Pediatr Surg. 2012;47:1555-9.

https://doi.org/IO.IoI6/j.jpedsurg.20I2.oI.oI4

13. Núñez-Cerezo V, Romo-Muñoz M, Amesty-Morello M, Vilanova-Sánchez A, Dore-Reyes M, Gómez-Cervantes A et al. Sistema de presión negativa en el tratamiento de fístulas enterocutáneas en la población pediátrica. Cir Pediatr. 2016;29:I66-I70.

I4. Liu J, Hu F, Tang J, Tang S, Xia K, Wu S et al. Homemade-device-induced negative pressure promotes wound healing more efficiently than VSD-induced positive pressure by regulating inflammation, proliferation and remodeling. Int J Mol Med. 20I7;39:879-88.

https://doi.org/IO.3892/ijmm.2017.2919

I5. Masumoto K, Nagata K, Oka Y, Kai H, Yamaguchi S, Wada M, et al. Successful treatment of an infected wound in infants by combination of negative pressure wound therapy and arginine supplementation. Nutrition. 20II;27:II4I-5.

https://doi.org/IO.IOI6/j.nut.20II.0I.006

I6. De Jesús LE, Martins AB, Oliveira PB, Gomes F, Leve T, Dekermacher S. Negative pressure wound therapy in pediatric surgery: How and when to use. J Pediatr Surg. 2018;53:585-91.

https://doi.org/IO.IOI6/j.jpedsurg.20I7.II.048

I7. Thomas S, Kriplani D, Crane C, Dehom SO, Oei G, Baerg J, et al. Outcomes in pediatric patients with abdominal compartment syndrome following urgent exploratory laparotomy. J Pediatr Surg. 2017;52:II44-7. https://doi.org/Io.IoI6/j.jpedsurg.2016.09.07I

I8. Arca MJ, Somers KK, Derks TE, Goldin AB, Aiken JJ, Sato TT, et al. Use of vacuum-assisted closure system in the management of complex wounds in the neonate. Pediatr Surg Int. 2005;2I:532-5. https://doi.org/I0.I007/s00383-005-I465-y

I9. Pauniaho SL, Costa J, Boken C, Turnock R, Baillie CT. Vacuum drainage in the management of complicated abdominal wound dehiscence in children. J Pediatr Surg. 2009;44:736-40. https://doi.org/IO.IOI6/j.jpedsurg.2009.0I.009

20. Ribeiro MA, Barros EA, Carvalho SM, Nascimiento VP, Cruvinel JN, Fonseca AZ. Comparative study of abdominal cavity temporary closure techniques for damage control. Rev Col Bras Cir. 20I6;43:368-73.

https://doi.org/I0.I590/oI00-699120160050I5

2I. Loftus TJ, Jordan JR, Croft CA, Smith RS, Efron PA, Mohr AM, et al. Temporary abdominal closure for trauma and intra-abdominal sepsis: Different patients, different outcomes. J Trauma Acute Care Surg. 20I7;82:345-50. https://doi.org/IO.I097/TA.ooooooooooool283

22. García-González M, Casal-Beloy I, Dovigo A, Miguez-Fortes L, Dargallo-Carbonell T, Pita-Fernández $\mathrm{S}$, et al. Negative pressure wound therapy for a complicated abdominal laparotomy in neonatal necrotizing enterocolitis: A case report. Ostomy Wound Manage. 20I7;63:34-8.

23. Rentea RM, Somers KK, Cassidy L, Enters J, Arca MJ. Negative pressure wound therapy in infants and children: A single-institution experience. J Surg Res. 2013;I84:658-64. https://doi.org/IO.IOI6/j.jss.2013.05.056

24. Baharestani M, Amjad I, Bookout K, Fleck T, Gabriel A, Kaufman D et al. V.A.C. therapy in the management of paediatric wounds: Clinical review and experience. Int Wound J. 2009;6(Suppl.I):I-26. https://doi.org/IO.IIII/j.I742-48IX.2009.00607.X

25. Birke-Sorensen H, Malmsjo M, Rome P, Hudson D, Krug E, Berg L, et al. Evidence-based recommendations for negative pressure wound therapy: Treatment variables (pressure levels, wound filler and contact layer) - steps towards an international consensus. J Plastic Reconstr Aesthet Surg. 20II;64(Suppl.):SI-Si6. https://doi.org/Io.IoI6/j.bjps.20II.06.ooI

26. de Leon JM, Barnes S, Nagel M, Fudge M, Lucius A, García B. Cost-effectiveness of negative pressure wound therapy for postsurgical patients in long-term acute care. Adv Skin Wound Care. 2009;22:122-7. https://doi.org/IO.I097/or.ASW.0000305452.79434.d9

27. Jiménez CE. Terapia de presión negativa: una nueva modalidad terapéutica en el manejo de heridas complejas, experiencia clínica con 87 casos y revisión de la literatura. Rev Colomb Cir. 2007;22:209-24.

28. Bautista W, Rodríguez D, Sáenz L. Uso de los sistemas de presión negativa en el tratamiento de infecciones asociadas a dispositivos: "una vieja terapia con un uso novedoso". Rev Colomb Cardiol. 20I7;24:57.eI-6. https://doi.org/IO.IoI6/j.rccar.2016.0I.027

29. Velásquez M, Garzón J, Martínez S. Cierre asistido con succión en heridas complejas de la pared del tórax. Rev Colomb Cir. 2009;24:185-98. 